\title{
Parameters identification of a time-varying stochastic dynamic systems using Viterbi algorithm
}

\author{
Tarik Al-Ani, Yskander Hamam \\ Ecole Supérieure d'Ingénieurs en Electrotechnique et Electronique \\ (E.S.I.E.E.), Département Automatique \\ Cité Descartes 2 bd Blaise Pascal - B.P. 99, 93162 Noisy-le-Grand \\ Cedex. e-mail: alanit@esiee.fr, hamamy@esiee.fr
}

\begin{abstract}
An on-line non-linear parameter identification algorithm is introduced. The system parameters are considered to be time varying. This algorithm is based on Viterbi algorithm applied to finite state models. It allows the identification based on the dynamic continuous output of the system. The algorithm is first applied to first order dynamic systems, then it is extended to a higer order systems. In order to reduce the complexity of the parameters discretisation, these parameters are supposed to be decoupled with respect to noise. Preliminary Simulation results are given.
\end{abstract}

Keywords

Stochastic process, hidden Markov chain, Stochastic dynamic programming, Parameter identification.

\section{INTRODUCTION}

In adaptive control of Time varying dynamic systems, on-line identification is a fundemental step. The problem of on-line identification of stochastic time varying dynamic systems has been extensively studied in the literature, Goodwin and Sin (1984), Isermann et al. (1992). The identification algorithms, in general, are based on either a state space models or the input-output models. In this paper, we adopt the input-output stationnary linear or non-linear models such as the Linear Autoregressiv model LARX and the Nonlinear Autoregressive model NARX. This choise is justified by the fact that these models are more convenient in practice and that the number of parameters to be estimated is resonable. Our approach is based on the Viterbi algorithm developped for decoding convolutional codes, Viterbi (1979), Forney (1972). This recursive algorithm is optimal in the sense that it is the maximum-likelihood estimation of the entire received observation sequence, Forney (1973). It applies the dynamic programming principle to the detection of discrete-time finite state Markov processes with noisy observations. This algorithm, which 
may be obtained by learning, uses a Hidden Markov Model (HMM), Rabiner (1989), to incorporate nonstationarity into input-output model. Thus our aim is to estimate time varying parameters in the LARX or NARX models. However, in this paper, the HMM is calculated on-line without learning.

The Viterbi algorithm is largely applied in communication theory, e.g. in convolutional coding, intersymbole interference, Viterbi and Omura (1979) and other domains such as signal processing, Streit and Barrett (1990). Furthermore, its computational complexity is linear with respect to the length of the measurment sequence.

As mentioned by Rabiner (1989), in a similar manner to the Kalman filter, the Viterbi algorithm tracks the states of stochastic processes with a recursive method that is optimum in a certain sense, and that lends itself readily to implementation and analysis. However, the underlying hidden process is assumed to be finite-state Markovian rather than Gaussian, which leads to marked difference in structure.

In order to apply the Viterbi algorithm, we proceed by descretizing the parameters spaces into $\mathrm{N}$ subspaces. Each subspace is then assigned its center to represent the hidden parameter value at time $\mathrm{k}$. The aim of this paper is to estimate the parameter sequence using the available measurment sequence.

The system models are defined in section (2). Section (3) describes the Viterbi algorithm for a single and multiple parameter dynamic systems. Simulation results are given in section 4. The conclusions of this paper are presented in section (5).

\section{SYSTEM MODELS}

Let us generally define the following discrete Single Input Single Output NARX model

$y(k)=\phi(\Theta(k) ; y(k-1), \ldots, y(k-p) ; u(k-1), \ldots, u(k-q))+v(k), k=0,1,2, \ldots, K,(1)$

where

$\Theta(k)=\left[\theta_{1}(k), \ldots, \theta_{p+q}(k)\right]^{\prime}$.

In LARX model, $\phi$, is defined by

$$
\begin{aligned}
\phi(.) & =\sum_{l=1}^{p} \alpha_{l}(k) y(k-l)+\sum_{l=1}^{q} \beta_{l}(k) u(k-l)+v(k), \\
\Theta(k) & =\left[\begin{array}{llll}
\alpha_{1}(k) \ldots \alpha_{p}(k) \beta_{1}(k) \ldots \beta_{q}(k)
\end{array}\right]^{\prime}
\end{aligned}
$$

where $y(k)$ is the output, $u(k)$ the input, $\alpha .(k), \beta .(k)$ are the model parameters which may be time dependent and subject to zero mean white stationnary disturbance

$$
\mathbf{w}(\mathbf{k})=\left[\begin{array}{llll}
w_{1} & w_{2} & \ldots & w_{p+q}
\end{array}\right]^{\prime}
$$

$\mathbf{E}\left(\mathbf{w}(k) \mathbf{w}^{\prime}(k)\right)=\mathbf{\Sigma}_{\mathbf{w}}, k=0,1,2, \ldots, K$.

In this paper, we assume that the the components of the disturbance vector in equation (3) are decoupled (i. e. $\boldsymbol{\Sigma}_{\mathbf{w}}$ is diagonal). The measurement noise $v(k)$ is considered to be a zero 
mean stationnary Gaussian random sequence, i.e $E(v(k))=0$ and $E\left(v(k)^{2}\right)=\sigma_{v}^{2}, k=$ $0,1,2, \ldots, K$. These errors are also considered to be uncorrelated with disturbance input. The initial parameters $\theta .(0)$ are considered to be a priori known. They may be constants or Gaussian random variables with mean values $m_{\theta(0)}$ and variance $\sigma_{\theta(0)}^{2}$.

\section{THE VITERBI ALGORITHM}

Based on dynammic programming methodes, this algorithm searches for a single best state (parameter) sequence (hidden Markov chain states). As mentioned in section (3.1), the underlying hidden process is assumed to be finite-state Markov. Thus we proceed by describing a Markov finite-state model for single parameter and then extend the results to Multiple parameter.

\subsection{Finite-space model for single parameter}

Let us denote the parameter value at time $\mathrm{k}$ by $\theta(k)$ and suppose that the allowed bounds of $\theta$ are known a priori, i.e. $S: \theta_{\min } \leq \theta(k) \leq \theta_{\max }$. This range is quantized into a finite number $\mathrm{N}$ of state cells $s_{i} \unrhd\left[\theta_{i}, \theta_{i+1}\right]$, i.e. $S=\bigcup_{i=1}^{N} s_{i}$ and each cell is associated with a state of the Markov chain. Each state $s_{i}$ of the Markov chain is represented by the cell center point $\theta_{c i}=\frac{\left(\theta_{i}+\theta_{i+1}\right)}{2}, i \in\{1,2, \ldots, N\}$, where $\theta_{1}=\theta_{\min }$ and $\theta_{N+1}=\theta_{\max }$. Let the state of the Markov chain at time $\mathrm{k}$ be $q_{k}$, if $q_{k} \in s_{i}$ then the estimated parameter at time $\mathrm{k}$ is $\hat{\theta}(k)=\theta_{c i}, i \in\{1,2, \ldots, N\}$.

\section{Single state Viterbi algorithm}

To find the single best Markov chain state sequence $\mathcal{F}_{k}^{s} \unrhd\left\{q_{1}, q_{2}, \ldots, q_{K}\right\}, q_{k} \in s_{i} \in \mathbf{R}^{\mathbf{1}}, i \in$ $\{1,2, \ldots, N\}$ for the given observation sequence $\mathcal{F}_{k}^{y} \unrhd\{y(1), y(2), \ldots, y(k)\}$ we define the best high score (highest probability) along a single path, at time $\mathrm{k}$, which accounts for the first $\mathrm{k}$ observations and ends in state $s_{i}$

$\delta_{k}(i)=\left[\max _{\mathcal{F}_{k-1}^{S}} \operatorname{Pr}\left(q_{k}=s_{i}, \mathcal{F}_{k}^{y} \mid \lambda\right)\right]$.

The parameter $\lambda$ is called in the literature "Hidden Markov Model (HMM)", Rabiner (1989). This model is defined by the triplet $\lambda \unrhd\{\boldsymbol{\Pi}, \mathbf{A}, \mathbf{B}\}$, where $\boldsymbol{\Pi}, \mathbf{A}$ and $\mathbf{B}$ are the Initial state distribution, state transition probabilities and measurement probability distribution, respectively.

$$
\begin{array}{ll}
\boldsymbol{\Pi}=\left[\pi_{1}, \pi_{2}, \ldots, \pi_{N}\right], & \pi_{i}=\operatorname{Pr}\left(q_{0} \in s_{i}\right), \\
\mathbf{A}=\left[a_{i j}\right], & a_{i j}=\operatorname{Pr}\left(q_{k+1} \in s_{j} \mid q_{k} \in s_{i}\right), \\
\mathbf{B}=\left[b_{j}(y(k))\right], & b_{j}(y(k))=\operatorname{Pr}\left(y(k) \mid q_{k} \in s_{j}\right),
\end{array}
$$

and $i, j \in\{1,2, \ldots, N\}, k \in\{0,1,, \ldots, K\} . y(k)$ may be, in general, discrete $y(k)=$ $V(k), V(k) \in\{1,2, \ldots, M\}$ or continuous $y(k) \in \mathbf{R}^{r}$ which is the case in this paper. The model $\lambda$ may be obtained off-line by training, Rabiner (1989). However, in our approach, training is not used. The parameters are directly calculated (off and on-line) using the observation model, equation (1). By induction on equation (3), the complete Viterbi 
algorithm may now be stated (using the natural logarithm to avoid data underflow) as follows

1. Initialization, $k=0$

$$
\begin{aligned}
\delta_{1}(i) & =\ln \pi_{i}+\ln b_{i}(y(1)), 1 \leq i \leq N, \\
\Psi_{1}(i) & =\mathbf{0}
\end{aligned}
$$

2. Recursion $1 \leq k \leq K$

$$
\begin{aligned}
\delta_{k}(j) & =\max _{1 \leq i \leq N}\left[\delta_{k-1}(i)+\ln a_{i j}\right]+\ln b_{j}(y(k)), 1 \leq j \leq N, \\
\Psi_{k}(j) & =\arg \max _{1 \leq i \leq N}\left[\delta_{k-1}(i)+\ln a_{i j}\right], \quad 1 \leq j \leq N,
\end{aligned}
$$

3. Termination

$$
\begin{aligned}
\ln P^{*} & =\max _{1 \leq i \leq N}\left[\delta_{K}(i)\right], & & \text { (Viterbi score), } \\
q_{K}^{*} & =\arg \max _{1 \leq i \leq N}\left[\delta_{K}(i)\right], & & \text { (Viterbi track), }
\end{aligned}
$$

4. Path (optimal state sequence) backtracking $k=K-1, K-2, \ldots, 0$

$$
q_{k}^{*}=\Psi_{k+1}\left(q_{k+1}^{*}\right),
$$

The state sequence may be restrained by keeping track of the argument which maximizes equation, for each $\mathrm{k}$ and $\mathrm{j}$. This is done using the array $\Psi_{k}(j)$. Thus the Viterbi algorithm may be used as a filtering as well as a smoothing algorithm. It should be noted that a treillis structure, efficiently implements the computation of the Viterbi algorithm, Forney (1973).

\section{Deriviation of the parameters of hidden Markov chains}

The two parameters $\boldsymbol{\Pi}$ and $\mathbf{A}$ may be calculated off-line. However the parameter $\mathbf{B}$ must be calculated on-line.

1. Initial parameter distribution, $\Pi$. The choice of $\pi$ depends, in general, on the application. In control applications, the initial parameter of the dynamic system or its expected value is, in general, known a priori. Thus, without loss of generality, we take $\pi_{i}=1, q_{0} \in s_{i}, i \in\{1,2, \ldots, N\}, \pi_{j}=0, j \in\{1,2, \ldots, N\} \neq i$.

2. State transition probabilities, $\mathbf{A}$. The definition of the state transition probability $a_{i j}, i, j \in\{1,2, \ldots, N\}$, is similar to that proposed by Streit and Barrett (1990). Based on the finite-space model discussed in section 3.1, we calculate, off or on-line, the state transition probabilities $a_{i j}$. If the state track lies in the ith cell at the current time step, the location of the track at the next time step is assumed to be characterized by a Gaussian distribution with mean $x_{c i}$ and standard deviation $\sigma_{w}$. Hence, the transition probability that the state shifts from the ith cell to jth cell at the next time step is

$$
a_{i j}=\frac{g_{i j}(k)}{\sum_{j=1}^{N} g_{i j}(k)},
$$




$$
g_{i j}(k)=\left(2 \pi \sigma_{w}\right)^{-\frac{1}{2}} \int_{\theta_{j}}^{\theta_{j+1}} \exp \left\{-\left[\frac{\left(\theta(k+1)-\theta_{c i}(k)\right)^{2}}{2 \sigma_{w}{ }^{2}}\right]\right\} d \theta
$$

$1 \leq i \leq N, 1 \leq j \leq N . \sigma_{w}$ represents the standard deviation of the pertubation $w(k)$.

3. The measurement probabilities, $\mathbf{B}$. The observation $y(k)$ in equation $(1)$ is a linear function of the measurement Gaussian noise $v(k)$. Hence, the conditional probability density function of $y(k)$, given that $\theta(k)=\theta_{c j}$, is a Gaussian density function

$$
b_{j}(y(k))=\left(2 \pi \sigma_{v}\right)^{-\frac{1}{2}} \exp \left\{-\left[\frac{\left(y(k)-\phi\left(\theta_{c j} ; y ; u_{.}\right)\right)^{2}}{2 \sigma_{v}^{2}}\right]\right\}, 1 \leq j \leq N
$$

Equation (6) must be evaluated for all states (parameter levels) $\theta_{c j}, j=1,2, \ldots, N$.

\subsection{Finite-space model for Multiple parameter dynamic system}

In order to apply the Viterbi algorithm to multiparameter system identification, we modify the single state Viterbi algorithm. The idea is similar to that proposed by Xie and Evans (1991) for multiple frequency tracking. We consider the system described by equations (1)(2). As mentioned in section 2 , the parameters $\theta_{l}(k), l=1,2, \ldots, p+q$, in equation (2) are decoupled with respect to perturbation noise (i. e. $\boldsymbol{\Sigma}_{\mathbf{w}}$ is diagonal). In this case, each parameter $\theta_{l}(k), l=1,2, \ldots, p+q$ is represented by a finite space model similar to that defined in section 3.1. The parameters $\theta_{l}(k), l=1,2, \ldots, p+q$, in equation (1) are decoupled with respect to perturbation noise. Thus, the hidden Markov parameters for each state may be derived in the same way as in section 3.1 .

\section{COMPUTER IMPLEMENTATION AND SIMULATION}

The Viterbi algorithm, conditioned on the whole past history of the possible parameters sequence, gives an optimum result. However, in practics this requires a large memory and long computation. Hence, the Viterbi algorithm is implemented in a suboptimum scheme in order to use a constant memory and achieve computation reduction. This scheme is based on a finite delay (defined by the memory). In order to keep a good performance, simulation or transfer function based approaches are used to evaluate this delay. Preliminary simulation has been carried out on PC using Matlab. The simulated ARX model used for the tests is

$y(k)=a_{1} y(k-1)+a_{2} y(k-2)+b u(k)+v(k)$,

where, $a_{1} \in\{1.6,1.7,1.8\}, a_{2} \in\{0.7,0.8,0.9\}$ and $b \in\{0.9,1.0,1.1\}$. For each simulation, the observation noise $\mathrm{v}(\mathrm{k})$ and disturbance noise $\mathrm{w}(\mathrm{k})$ were taken to be stationnary white Gaussian with 0.1 standard deviation. The input signal u was generated at random. Figure 1 clearly shows that the Viterbi identifier works well. The accuracy may be increased by increasing the number of the discretization levels. 


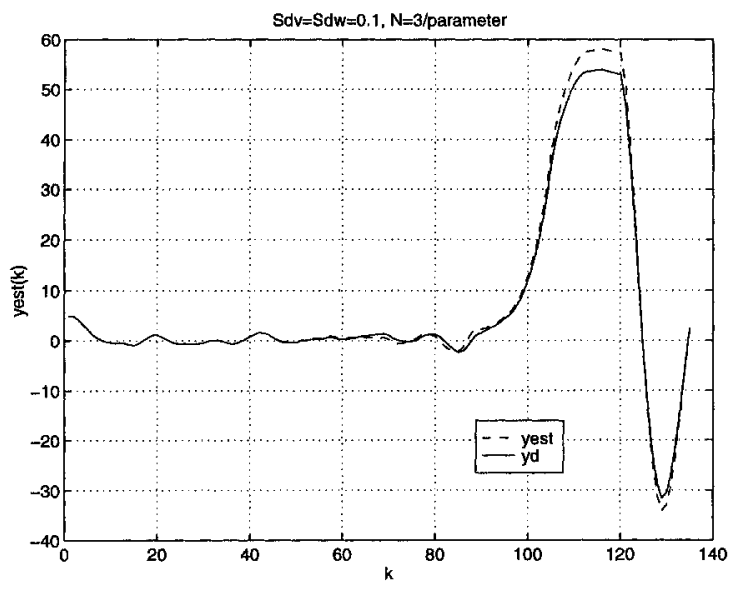

Figure 1 Computer simulation.

\section{CONCLUSION AND PERSPECTIVES}

In this paper, the application of the Viterbi algorithm to parameter identification of a general (linear or non-linear) dynamic systems has been described. The following conclusions may be drawn.

- Simulation has shown that the Viterbi algorithm may be used to identify time varying dynamic systems.

- The identification accuracy may be improved by increasing the discretization levels.

- The Viterbi algorithm allows discrete parameter identification depending on the cell resolution. To overcome this difficulty, continuous parameter identification may be obtained using the conditional average of the states, Streit and Barrett (1990).

- An on-line learning scheme of the Hidden Markov chain may considerably increase the accuracy of the identification.

The authors are presently working on the extensions of the above work to cover both continuous parameter representation and an on-line recusive extensions.

\section{REFERENCES}

Goodwin, G.C. and Sin, K.S. (1984) Adaptive Filtering, Prediction and Control. PrenticeHall.

Forney, G.D. (1973) Maximum-Likelihood sequence estimation of digital sequences in the presence of intersymbol inference. IEEE Trans. Inform. Theory, 18, 363-78.

Forney, G.D. (1973) The Vitrbi algorithm. Proc. IEEE, Mars, 268-78. 
Isemann, R., Lachmann, K.-H. and Matko, D. (1992) Adaptive Control Systems. PrenticeHall.

Rabiner, L.R. (1989) A tutorial on hidden Markov Models and selected applications in speech recognition. Proc. IEEE, 77, 257-86.

Streit, R.L. and Barrett, R.F. (1990) Frequency line tracking using Hidden Markov Models. IEEE Trans. Acoust., Speech, Signal Processing, 38, 586-98.

Viterbi, A.J. (1967) Error bounds for convolutional codes and asymptotically optimum decoding algorithm. IEEE Trans. on Information Theory, IT-13, 260-9.

Viterbi, A.J. and Omura, J.K. (1979) Principles of Digital Communication and Coding. McGraw-Hill, New York.

Xie Xianya and Evans Robins J. (1991) Multiple Target Tracking and Multiple Frequency Line tracking Using Hidden Markov Models. IEEE Trans. on Signal Processing, 39, $2659-76$. 